\title{
Involving consumers in assessing service quality: benefits of using a qualitative approach
}

\author{
Jackie Powell, Robin Lovelock, Jane Bray, Ian Philp
}

\begin{abstract}
Although important to users, practice standards rarely incorporate users' views of care provided. These views are a valuable source of information, even though there are limits to their value. To improve the standards of care in a 20 bed hospital elderly care unit caring for acute medical conditions a qualitative approach was used. Patients' and carers' perceptions of care and problems with the process of care in the unit were elicited with a specially designed semistructured interview schedule in 83 separate tape recorded interviews with a research nurse in patients' homes. In all, 50 patients and 35 carers were interviewed between 6 June 1991 and 28 May 1992. Of the 50 patients, 33 were female; seven patients were aged less than 80 years, $16,80-85 ; 21,86-90$; and six over 90 . A total of 16 patients lived with spouses or other carers, two with noncarers, and 32 lived alone, 18 of whom received informal care. Content analysis of the interviews disclosed patients' and carers' general satisfaction with individualised professional care and planning of follow up services on discharge but dissatisfaction in the lack of information about and involvement in treatment and care and about specific staff notes. These findings have prompted remedial changes in clinical practice in the unit; they have also formed the structure of a criterion based survey of practice. The authors conclude that the qualitative approach suited elderly users and also provided the basis for the findings to be incorporated into a continuous audit cycle through a process of feedback and standard setting. (Quality in Health Care 1994;3:199-202)
\end{abstract}

\section{Introduction}

Improving the quality of health care offered to the public is now central to National Health Service policy in the United Kingdom (NHS and Community Care Act 1990). Both managers and practitioners support this view, and most hospitals and many general practices have clinical audit programmes. A growing number of important initiatives are focusing on quality issues and ways of improving standards in health care. However, other views of quality need to be included within the specification of standards. Standards of practice are crucial to the users of services, but users are rarely involved in the process of establishing them. Although there are limitations to the value of users' views for service evaluation, ${ }^{1}$ they are a valuable source of information about experience of treatment.

Recognising the need for more consumer involvement, the Patient and Carer Satisfaction (PACS) Group was established in April 1991. The group included representatives from Southampton University's departments of geriatric medicine and social work studies and members of the service planning department of the district health authority and management and staff of the elderly care unit. This formed one part of an overall quality strategy for the elderly care unit at Southampton General Hospital. The studies initiated by this group were designed to give particular prominence to the consumer viewpoint in caring for older people. A qualitative approach to obtaining the data was used, giving priority to flexibility in covering topics, clarification of ambiguities, and sensitivity to patients' concerns. ${ }^{2}$ Although conceived as valuable research findings in themselves, ${ }^{3}{ }^{4}$ these results were also seen as contributing to the audit cycle through the process of feedback to staff and setting standards.

The aims of the study were, firstly, to examine patients' perceptions of care in the unit, taking into account carers' views of the effectiveness of the service provided and, secondly, to identify problems with the process of care, especially discharge planning, and to provide feedback to staff to enable changes in practice to be considered.

\section{Methods}

An audiotaped group discussion with nurse managers and medical staff of the elderly care unit was set up to elicit their concerns about providing inpatient care and planning for discharge. This discussion, with documentary data and our own observations, provided the basis for the design of a detailed interview schedule for use (in slightly varying formats) with both patients and carers. An interview schedule, rather than a structured questionnaire, was used as an aide memoire for the interviewer, setting out and linking the main themes and subsidiary topics identified for exploration, and providing an overall framework for each interview. The main topics covered were admission, ward setting, staff contacts, treatment and care, discharge, and 
community services follow up. Within each broad topic more detailed aspects or topics were listed.

This study focused on one 20 bed ward caring for older people with acute medical conditions. Most patients are admitted through their general practitioner as medical emergencies. Alternate patients discharged from this ward to the community (that is, to a non-institutional setting) were asked whether they would agree to be interviewed and whether their carer could be approached. We defined a carer as a person (family or friend) who gives some informal input of care or support in the patient's home. The interviews were conducted in patients' homes by one research nurse (JB) two to four weeks after their discharge. The main data collection phase of the research took place between 6 June 1991 and 28 May 1992.

By adopting a conversational style, respondents were encouraged to recount their experiences and views of the service in a relatively free and unprompted manner. To facilitate this process each interview took the form of a tape recorded discussion between the patient or carer and the research nurse. Transcripts of the interviews were produced with a simple word processing package. They were then categorised and ordered in a preliminary way using the main topics of the interview schedule and the "cut and paste" facility of the word processing package. Thus the main issues explored in the interviews also provided a structure for the first stage of analysing users' and carers' views.

JB and JP were actively involved in the analysis of the data, which entailed becoming familiar with the data through the process of reading, ordering, and re-reading the individual accounts of people's experiences and comparing these accounts according to the topics previously identified in the interview schedule. The process of analysis and interpretation entailed both self reflexivity and continuous checking of the procedures used in presenting the results and selecting illustrative quotations. The final organisation of the data substantially reflected the prior framework of topics but also took account of clusters of views and issues emerging from the interviews themselves and distilled through the process of analysis.

The findings were compiled as a written report, in which items of concern in patient care and possible actions were highlighted. Our discussion of these findings was not intended to be highly prescriptive, but was offered as a basis for wider discussion among those people more closely concerned with the day to day task of managing and providing the service.

\section{Results and discussion}

All 50 of the patients identified for inclusion in the study were interviewed: 33 were female and 17 were male. Seven patients were aged less than 80 years, 16 between 80 and 85,21 between 86 and 90 , and six over 90 (mean age 85.04 years; median age 86 years). Sixteen patients lived with spouses or other carers, and two lived with people identified as not having the role of carer. Thirty two patients lived alone, of whom 18 received some informal help from either family or friends. In total, 83 separate interviews were carried out involving 50 patients and 35 carers. These included two joint interviews.

The table summarises the key findings from the analysis of the interviews.

\section{ADMISSION}

All patients interviewed thought that their admission had been appropriate, a view echoed by their carers, many of whom had experienced feelings of relief. As most of the patients were admitted to the unit as emergencies through their general practitioner their physical wellbeing had been the primary concern for them and their carers. The need for information at this time was not seen as a priority, although any friendly reassuring words were greatly appreciated. Written information, when available, was seen as helpful.

These findings suggest that although patients and carers valued information about the ward and the unit as a whole, they were not always able to absorb it at the time of the admission. Once the immediate crisis has passed, written information might prove helpful as an ongoing resource and reference point. This could include the names of the professional team caring for the patient and emphasise the specialist nature of the ward in terms of providing care for older people. Very few patients seemed to understand the primary nursing system, although carers seemed more aware of this and appreciated the possibility of an identified point of contact. The system could have been made more explicit in written information to patients and carers and in daily oral reminders.

PROFESSIONAL CARE

Patients, and to some extent their carers, seemed to have limited knowledge about their medical treatment. Some patients felt no need for information, expressing trust in those whom they perceived as the professional experts. When patients had asked for information they were satisfied with what they had been told. Other patients and their carers would have welcomed further explanation, although they had not actively sought additional information.

Summary of key findings

\begin{tabular}{|c|c|c|}
\hline Category & Positive comments & Critical comments \\
\hline General & Individualised care & $\begin{array}{l}\text { Lack of information } \\
\text { Lack of involvement in decision making }\end{array}$ \\
\hline $\begin{array}{l}\text { Admission } \\
\text { Professional care } \\
\text { Drug treatment } \\
\text { Discharge planning } \\
\text { Follow up }\end{array}$ & $\begin{array}{l}\text { Appropriate } \\
\text { Trust in the professionals } \\
\text { Guidance from nursing staff (minority of cases) } \\
\text { Carers involved (minority of cases) } \\
\text { Services materialised as expected }\end{array}$ & $\begin{array}{l}\text { Lack of information (for example, role of primary nurse) } \\
\text { Lack of involvement in planning care } \\
\text { Confusion when drugs changed } \\
\text { Lack of involvement of patients } \\
\text { Lack of integration of formal and informal support }\end{array}$ \\
\hline
\end{tabular}


How much people wanted to know varied considerably, although it seems important to allow both patients and carers opportunities to become more involved in discussing treatment and future care. The primary nurse is in the best position for getting to know a patient well and may therefore be able to judge how this might be addressed individually. Closer liaison between the primary nurse and medical staff might also facilitate this process. The pivotal role of the nurse for providing information to patients and the need for close liaison between nursing and medical staff has been identified in another, albeit small, study of elderly patient satisfaction with hospital care. ${ }^{5}$

DRUG TREATMENT

Overall, patients' and carers' comments suggested that they were not well informed about drug treatment; some patients and carers reported confusion when drug treatment had been changed, particularly if they felt that no adequate explanation had been offered. In these situations, once a patient was discharged the general practitioner was the most likely person to be seen as the key point of reference.

In the small number of instances in which patients had been given particular help in understanding and administering their own drugs, mainly by nurses, this had been appreciated by patients and carers. Written information about the treatment had been given and time had been spent with patients and carers explaining the treatment. Wider adoption of these practices could clearly benefit many more patients and their carers.

\section{DISCHARGE PLANNING}

Patients' comments about discharge centred around their experience of being told when they would go home. They made little reference to any discussion that they might have had about how they were to manage at home once discharged. Unless patients were very vocal, they did not seem to be involved in the decision making. Some carers referred to their having had some discussion with ward staff about the possibility of various forms of follow up help, and in these situations they seemed aware of the complexities of arranging follow up services.

Given these comments and the unit's stated philosophy of providing an approach centred on people, the health professionals need to consider ways of more actively involving the patient and, where appropriate, the carer in discharge planning. Developing a system, possibly involving the primary nurse, which enables patients as well as their carers to become more aware of what follow up arrangements have been organised, could increase patient and carer participation in the decision making process.

FOLLOW UP

Most patients indicated that they had received the services they had expected, suggesting that the liaison between hospital staff and those providing community services had been successful. However, in a few cases, some difficulties had been encountered. Close liaison between ward staff and the various community services is crucial at all times if an adequate understanding of how each service works is to be achieved and effective communication maintained. Both patients' and carers' comments also disclosed the ways in which many patients had complex networks of support, which often involved a web of formal and informal sources of help. There seems to be a need for greater awareness and understanding of these systems and how, quite often, there is a fine balance between the patient maintaining independence in the community and the system breaking down. Professional inputs could be better focused on maintaining these often highly individualised support networks, rather than offering a relatively routine formal service.

\section{FEEDBACK}

The final report from the study ${ }^{3}$ was presented to the PACS group, who recommended that the findings should be developed by a multidisciplinary quality assurance group (convened by the PACS Group during the period of the study specifically to address standard setting and audit of services). As a result several changes were made in the unit as follows.

(1) An information leaflet was produced, outlining the facilities offered by the unit and describing the role of each member of the multidisciplinary team.

(2) Photographs of the staff are displayed in areas of the ward as a means of informing patients and carers about the different staff members.

(3) A programme has been implemented whereby patients are encouraged to take charge of their own treatment, and written information and instructions are being used more.

(4) A leaflet is being designed to outline individual plans for each patient's discharge from hospital and details of follow up care in the community.

The findings of this study have also been used to identify dimensions for standard setting. These areas have formed the structure of a criterion based survey of practice, ${ }^{6}$ thus creating a model for integrating information of user satisfaction with clinical audit (figure).

Overall, patients and carers were generally very satisfied with the services they had received. They were especially appreciative of the individualised care that had been provided by the ward staff. Nevertheless, they highlighted many of the concerns shared by service providers about, for example, the need to offer more information related to medical treatment and drug treatment and to involve patients in decisions related to their care and discharge.

The qualitative approach used in this study enables a more genuine "voice of the consumer" to be heard and taken account of in the development of services. ${ }^{7}$ This is particularly useful when seeking the views of elderly people, especially very old people, many of whom are frail. ${ }^{8} 9$ Often disabled by poor communication skills and low self esteem, older people commonly need to be encouraged 


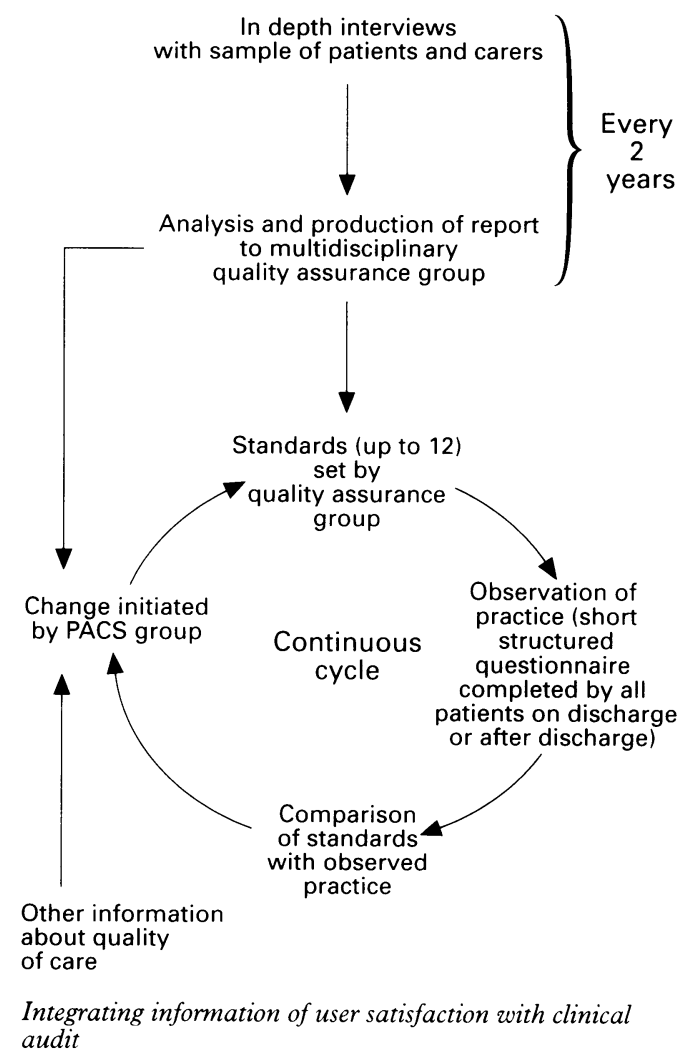

to express their views and opinions in a manner of their choosing, rather than be constrained through the use of a tightly structured questionnaire about specific aspects of the service. Using an interview schedule in the form of a checklist of themes and topics demands that the interviewer has sufficient command of the overall subject matter and possesses the skill of active listening, which has implications for training audit officers. ${ }^{10}$

If audit is to be effective the necessary investments of time and other resources must be made. ${ }^{11}$ The approach described here is demanding in these terms, but it has significant long term benefits: it provides a more authentic picture of service users' views and thus a more informed understanding of patients' and carers' concerns among service providers, strengthening the basis from which to develop service standards which can be incorporated into a continuous audit cycle.

1 Lebow J. Consumer satisfaction with mental health treatment. Psychol Bull 1982;91:244-59.

2 Fitzpatrick R. Surveys of patient satisfaction. I. Important general considerations. BMF 1991;302:887-9.

3 Powell J, Lovelock R, Bray J, Philp I. Patients' and carers'views on service quality (I). Southampton: CEDR, Department of Social Work Studies, University of

4 Powell J, Lovelock R, Bray J, Philp I. Patients' and carers' views on service quality (II): rehabilitation ward. carers views on service quality (II): rehabilitation ward.
Southampton: CEDR, Department of Social Work Southampton: CEDR, Department of

5 Morle K. Patient satisfaction: care of the elderly. $f A d v$ Nursing 1984;9:61-6.

6 Shaw C. Criterion based audit. BMF 1990;300:649-51.

Powell J, Lovelock R. The role of consumers' views in the evaluation of services: a case study - the travelling day hospital. Social Services Research 1987;16:16-29.

8 Macpherson I, Hunter DJ, McKeganey NP. Interviewing elderly people: some problems and challenges. Research, Policy and Planning 1988;4(2):13-8.

9 Powell J, Lovelock R, Bray J, Philp I. Quality issues in discharge from hospital - the views of older people and discharge from hospital - the views of older people
their carers. Social Services Research 1994;22:42-55.

their carers. Social Services Research 1994;22:42-55.
10 Firth-Cozens J, Venning P. Audit officers: what are they up to? BMF 1991;303:631-2

11 Shaw C, Costain D. Guidelines for medical audit: seven principles. BMF 1989;299:498-9. 